\title{
ON THE NATURE AND THE ORDER OF THE DECONFINING TRANSITION IN QCD.
}

\section{In honour of Yu. A. Simonov on his seventyth birthday.}

\author{
Adriano Di Giacomo ${ }^{1}$ \\ ${ }^{1}$ Dipartimento di Fisica dell'Università di Pisa , and INFN Sezione di Pisa \\ l.go Pontecorvo 2, I-56127, Pisa, Italy

\begin{abstract}
The determination of the parameters of the deconfining transition in $N_{f}=2$
\end{abstract} \\ QCD is discussed, and its relevance to the understanding of the mechanism of color \\ confinement.
}

\section{INTRODUCTION.}

Understanding the mechanism by which QCD confines colored particles is one of the most fundamental and challenging problems in field theory[1][2].

Experiments aimed to detect the existence of a deconfining transition at high temperature by colliding heavy ions have not yet produced a definite answer[3]. Up to now the deconfining transition has been only observed in numerical simulations of QCD on a lattice[4]. Identifying a signal of deconfinement is far from trivial in experiments, and is not easy in lattice simulations either. To be rigorous confinement means absence of colored particles in asymptotic states. Of course one cannot check confinement by looking at all asymptotic states. In the quenched approximation, in which dynamical quark loops are neglected, one looks at the static potential acting between a heavy $Q-\bar{Q}$ pair. The criterion for confinement is then the existence of a string tension, which means a linear behaviour at large distances

$$
V(r)=\sigma r
$$

The static potential is measured on the lattice either in terms of the Wilson loop (parallel transport along a closed path), or equivalently in terms of the correlator of Polyakov lines (parallel transports along the time axis). For a Wilson loop of extension $t$ in the time direction and $r$ in space

$$
W(r, t) \underset{r, t \rightarrow \infty}{\propto} \exp [-t V(r)]
$$


Confinement eq(1) implies the so-called area law

$$
W(r, t) \propto \exp [-\sigma r t]
$$

$r, t \rightarrow \infty$ means that they are large compared to the correlation length. The correlator of two Polyakov lines can be written, by use of cluster property,

$$
\left\langle L(r) L^{\dagger}(0)\right\rangle \underset{r \rightarrow \infty}{\simeq} C \exp [-\sigma r / T]+|\langle L\rangle|^{2}
$$

On the other hand

$$
V(r) \simeq-T \ln \left\langle L(r) L^{\dagger}(0)\right\rangle
$$

It is found by numerical simulations that a critical temperature $T_{c}$ exists such that for $T<T_{c}$ $\langle L\rangle=0$. From eq's(4) and (5) confinement eq(1) then follows. For $T>T_{c}\langle L\rangle \neq 0$ and

$$
V(r) \underset{r, t \rightarrow \infty}{\rightarrow} \text { const }
$$

For quenched $\left.S U(3) T_{c} / \sqrt{(} \sigma\right) \approx .65$ [5]. A deconfining phase transition exists at $T_{c} \simeq$ $270 \mathrm{Mev}$ and the Polyakov line $\langle L\rangle$ is the order parameter. The symmetry involved is $Z_{3}$ which is broken at $T>T_{c}$, and the transition is order-disorder. Some problem can arise in the continuum limit with the definition [6] of the order parameter $\langle L\rangle$, but the main idea looks sound.

The situation is less clear in the more realistic case of full QCD, including dynamical quarks. There $Z_{3}$ is not a symmetry anyhow, being broken by the coupling to the quarks. Moreover the potential at large distances is not expected to grow with $\mathrm{r}$, due to the conversion of the potential energy into light $q-\bar{q}$ pairs (string breaking)[7]. One needs then an alternative criterion for confinement, which ,however, is not known.

A phase transition is expected anyhow at low quark masses from the low temperature phase in which chiral symmetry is spontaneously broken, to a phase in which it is restored: the order parameter for this transition is the chiral condensate $\langle\bar{\psi} \psi\rangle$. This transition is indeed observed on the lattice at $T_{c} \approx 170 \mathrm{Mev}$. A priori restoration of chiral symmetry is not the same as deconfinement, even if it is physically understandable , e.g. by thinking of a bag model, that confinement can imply chiral symmetry breaking. In the absence of a criterion for confinement the question if chiral and deconfining transition coincide can not even be asked. A third symmetry exists, the axial $\mathrm{U}(1)$, which is broken at low $\mathrm{T}$ as an effect of the anomaly, and is also expected to be restored at high temperature. In 
principle the corresponding transition temperature could be different from that of the other two transitions.

An analysis can be done of the chiral transition based on symmetry arguments and on renormalization group techniques [8]. If the assumption is made that the relevant degrees of freedom at the transition are the pseudoscalar particles an effective lagrangean can be written on the basis of symmetry and scale invariance, which describes the density of free energy around the chiral point. For $N_{f} \geq 3$ no infrared stable fixed point exists, so that the transition is expected to be first order. $N_{f}=2$ is a special case, and the transition can either be weak first order or second order. If it is first order, it is weak first order also at $m_{q} \neq 0$; if it is second order the transition at $m_{q} \neq 0$ is a crossover [8]. What is the case can be investigated by numerical simulations on the lattice, even if up to now [9][10][11] the results have been rather elusive.

This issue is very important for the understanding of the deconfining phase transition. On the basis of the quenched case mentioned above the deconfining transition should be orderdisorder, and a genuine order parameter should exist labeling the two phases. $N_{c} \rightarrow \infty$ arguments suggest indeed that the symmetry involved as well as the mechanism of confinement should be the same for quenched and unquenched and $N_{c}$ independent. If for $N_{f}=2$ the chiral transition is second order, then the transition for $m_{q} \neq 0$ is a crossover, and the deconfining transition is not order-disorder. A first order chiral transition would instead be consistent with order-disorder, and possibly be such up to $m_{q}=\infty$, which is the quenched case.

The order of the transition can be investigated by looking at the volume dependence of the specific heat in numerical simulations, by a technique known as finite size scaling [see sect 2 below]. The free energy density around the phase transition is determined by symmetry arguments [12] up to unknown numerical coefficients, in terms of the order parameter. The (pseudo)critical indices determined by looking at the susceptibility of the order parameter must agree with the determination made by looking at the specific heat . Such an agreement is needed to legitimate the choice of the order paramenter.

An order parameter $\langle\mu\rangle$ has been developed and tested [13][14][15], based on the working hypothesis that the mechanism of confinement is dual superconductivity of the vacuum[2]. $\langle\mu\rangle$ is the vacuum expectation value of an operator $\mu$ carrying magnetic charge, and, unless the Polyakov loop or the chiral condensate is well defined independent of $N_{c}, N_{f}$ even in the 
continuum limit.

The critical indices can be investigated by measuring susceptibilities involving $\mu$, in particular the quantity $\rho=d / d \tau[\ln (\langle\mu\rangle)]$, with $\tau$ the reduced temperature

$$
\tau=1-T / T_{c}
$$

If they agree with the determination made by use of the specific heat, an additional legitimation results for the order parameter and for the mechanism of confinement by dual superconductivity. Confinement will then be defined in terms of an appropriate symmetry, and the question whether the deconfining transition and the chiral transitions occurr at the same temperature becomes meaningful.

In sect 2 we shall discuss new results on the above issues[16],[17] .

In sect3 we draw some conclusion.

\section{LATTICE RESULTS AND FINITE SIZE SCALING ANALYSIS.}

The theory of finite size scaling for higher order and weak first order phase transitions is based on renormalization group arguments [18][19], which are expected to hold when, by approaching the transition, the correlation lengths become much larger than the lattice spacing. It allows to extrapolate to infinite volume results obtained at finite volumes. The extrapolation depends on the (pseudo)critical indices, which can then be determined from the volume dependence, and with them the order and the universality class of the transition.

The relevant quantities, which are related to derivatives of the free energy, and hence to the order of the transition, are the susceptibilities. In the following we shall consider the susceptibility of the chiral order parameter

$$
\chi=\frac{T}{V} \frac{\partial^{2}}{\partial m^{2}} \ln Z
$$

the specific heat

$$
c_{V}=\frac{1}{V T^{2}} \frac{\partial^{2}}{\partial(1 / T)^{2}} \ln Z
$$

and

$$
\rho=\frac{\partial}{\partial \tau} \ln \langle\mu\rangle
$$

All these susceptibilities will depend on the parameters which describe the system, i.e. $\beta=\frac{2 N_{c}}{g^{2}}$ with $g$ the gauge coupling, and $m a$, the bare quark mass in units of the inverse lattice spacing. 
The simulation is made on a lattice $N_{t} N_{s}^{3}, N_{t} \ll N_{s}$, of extension $N_{t}$ spacings along the time axis and $N_{s}$ along the three spatial directions, with periodic boundary conditions (bc) in time for bosons,antiperiodic bc for fermions.

The temperature is the inverse of the time extension, or

$$
T=\frac{1}{N_{t} a(\beta, m a)}
$$

$a(\beta, m a)$ is the lattice spacing in physical units.

All the susceptibilities as function of the temperature will have peaks at $T_{c}$, which diverge as the volume $V \rightarrow \infty$ with a power depending on the order of the transition ,specifically on the critical indices. In the usual notation of statistical mechanics,denoting by L the spatial size of the system

$$
\begin{aligned}
\chi & =L^{\gamma / \nu} \Phi_{\chi}\left(\tau L^{\frac{1}{\nu}}, m L^{y_{h}}\right) \\
c_{V} & =c_{V}^{0}+L^{\alpha / \nu} \Phi_{c}\left(\tau L^{\frac{1}{\nu}}, m L^{y_{h}}\right) \\
\rho & =L^{\frac{1}{\nu}} \Phi_{\mu}\left(\tau L^{\frac{1}{\nu}}, m L^{y_{h}}\right)
\end{aligned}
$$

Since $T=\frac{1}{N_{t} a(\beta, m a)}$

$$
\tau=1-\frac{a\left(\beta_{c}, 0\right)}{a(\beta, m a)}
$$

In the vicinity of the chiral point one can expand in powers of $\beta_{c}-\beta$ and of $m a$, obtaining

$$
\tau \propto \beta_{c}-\beta+K m a
$$

with

$$
K=-\frac{\partial \ln a}{\partial(m a)} /\left.\frac{\partial \ln a}{\partial \beta}\right|_{\beta=\beta_{c}}
$$

In the quenched case the last term in eq(16) is absent.

When the volume of the system goes large at $\tau L^{\frac{1}{\nu}}$ fixed, the susceptibilities will tend to a finite limit certainly if the transition is second order, and the free energy is continuous and finite in the neighbourhood of the chiral point. This implies that the powers of $\mathrm{L}$ in front of the susceptibility eq's $(12),(13),(14)$ have to be eliminated by the dependence on $m L^{y_{h}}$, and this means for the three susceptibilities

$$
\begin{aligned}
\chi & \propto m^{-\frac{\gamma}{\nu y_{h}}} F_{\chi}\left(\tau L^{\frac{1}{\nu}}\right) \\
c_{V}-c_{V}^{0} & \propto m^{-\frac{\alpha}{\nu y_{h}}} F_{c}\left(\tau L^{\frac{1}{\nu}}\right) \\
\rho & \propto m^{-\frac{1}{\nu y_{h}}} F_{\rho}\left(\tau L^{\frac{1}{\nu}}\right)
\end{aligned}
$$


If the transition is weak first order the system will behave in the same way for intermediate values of L, smaller or equal than the critical correlation length, apart for the different value of the critical indices.

Eq's(18,(19),(20) imply that the maxima of the susceptibilities with respect to $\tau$ lie on the line of the plane $(\beta, m a)$ of equation

$$
\tau L^{\frac{1}{\nu}}=C
$$

with $C$ a constant, or, by use of eq(16)

$$
\beta_{c}-\beta+K m a-\frac{C}{L^{\frac{1}{\nu}}}=0
$$

This relation can be tested against the lattice data, to determine the value of $\nu$. We will discuss the result in the following .

Eqs (18),(19),(20) also imply that height of the the maximum of the susceptibilities is volume independent, whatever the order of the transition, in the limits of the regime in which these equations are obeyed when the transition is first order.

For second order transition the result is valid for any volume and fits the idea that the transition line in the plane $(\beta, m a)$ is a crossover.

For a first order transition, if we cross the transition line at fixed $m a$, we expect a behaviour typical of a first order transition, namely a growth of the peaks of the susceptibilities proportional to the volume. Indeed for a first order transition $\alpha=\gamma=1, \nu=1 / d=1 / 3$. This behaviour will be visible when L becomes larger than the critical correlation length, and together with it the typical two-peak distribution of the internal energy will appear.

Moreover, if we keep $m L^{y_{h}}$ constant, eqs $\left.(12),(13),(14)\right)$ require that the maximum of the peaks is again proportional to a well defined power of $\mathrm{L}$ depending on the nature of the transition.

The details of the simulations will be reported elsewhere [16][17] Here we only summarize the results.

As a strategy we assume the critical indices expected for second order $\mathrm{O}(4)$ phase transition and in alternative those of a first order transition and we investigate wether data are consistent with either of them. A measure of the agreement is the value of $\chi^{2} /$ dof.

In table 1 we recall values of the critical indices for the two cases.

We investigate 
1) The position of the peak as a function of mass and volume, which scales according to $\operatorname{Eq}(22)$. The $\chi^{2} / d o f$ is $\sim 1$ for the choice first order, and typically $\sim 10$ for $\mathrm{O}(4)$ second order.

2) The peak values of the susceptibilities. They all occurr on the same line, within errors. The height of the peak is volume independent at fixed $m a$ for moderate values of the volume and proportional to an appropriate power of ( $m a)$ Eqs(18)-(20), which depends on the nature of the transition. Here again the $\chi^{2} /$ dof is compatible with 1 for a first order transition, and much worse, typically [ $\left.\chi^{2} / d o f \approx 10\right]$ for $\mathrm{O}(4)$ second order.

3) For low values of $\mathrm{m}(\mathrm{e} . \mathrm{g} . \mathrm{ma}=.0135)$ as the volume increases further a growth of the height of the peak is observed and some sign of bistability in the time histories of the energy density. An increase of the heigth of the peak by a factor $\approx 2$ is observed going from $16^{3}$ to $32^{3}$. This means that there is a transition, and not a crossover. At higher values of $m a$ the transition looks weaker and we were not yet able to reach a large enough volume to see a bistability .

4) The behaviour with respect to volume at fixed $m L^{y_{h}}$ is consistent with first order transition, and disfavours second order : typically $\chi^{2} /$ dof $\approx 1$ for first order, $\chi^{2} /$ dof $\approx$ 10 for second order $\mathrm{O}(4)$.

Previous investigations of the same system were made on rather small volumes[10][9] [11] or in a less systematic way, and the results where admittedly inconclusive, although a slight psicological preference appeared for a second order transition . Numerically our determinations are consistent with the previous ones when done at the same values of the parameters. The details of the simulations and the comparison with previous work will be presented elsewhere [16][17].

\section{DISCUSSION.}

We have obtained substantial preliminary evidence that the chiral transition for $N_{f}=2$ QCD is first order. This makes the deconfining transition consistent with an order disorder

transition. The disorder parameter $\langle\mu\rangle$ detecting dual superconductivity of the vacuum provides a determination of the critical indices consistent with the one based on the specific 
heat,and a transition line consistent with the other susceptibilities. It can then be used as an order parameter for confinement. The deconfining transition and the chiral transition occurr at the same T. A careful numerical study of the U(1) axial anomaly across the deconfining transition is on the way and will provide a check of the assumption made in [8] that the relevant degrees of freedom at the chiral transition are the pseudoscalar goldstone particles.

I wish to thank all my collaborators, M. D'Elia, B.Lucini, G. Paffuti, C. Pica who contributed substantially to the results presented. Many ideas and motivations also come from discussions and collaboration with Yu. A. Simonov, to whom this paper is dedicated with deep friendship in the occasion of his 70th birthday. 
1. G.'tHooft, Nucl.Phys. B138, 1 (1978).

2. G.'tHooft, Nucl. Phys. B 190, 455 (1981).

3. Proceedings of the International Conference Quark Matter 2002 Nucl.Phys. A 715 (2003).

4. For a review see e.g. F.Karsch, Nucl. Phys. B(Proc.Suppl) 83-84, 14 (2000).

5. Y.Iwasaki et al. Phys. Rev. D 56, 151 (1997).

6. O. Kaczmarek, F.Karsch, P.Petreczky, F.Zantow, Phys. Lett. B 543, 41 (2002)

7. See e.g. C.De Tar, O.Kaczmarek, F.Karsch, E,Laerman, Phys.Rev. D 59, 031501, (1999), and references therein.

8. R.D. Pisarsky and F. Wilczek, Phys. Rev. D 29338 (1984).

9. F. Karsch, E. Laerman, Phys. Rev. D 50, 6954, (1994).

10. S.Aoki et al., JLQCD Collaboration, Phys. Rev. D 57, 3910 (1998)

11. C. Bernard et al. Phys. Rev. D46, 4741 (1992)

12. V.L. Ginzburg,L.D. Landau, Zh.Eksp.Teor.Fiz.20, 1064 (1950).

13. L.Del Debbio, A. Di Giacomo, G. Paffuti, G. Pieri, Phys. Lett. B355, 255 (1995).

14. A. Di Giacomo, B. Lucini, L. Montesi, G. Paffuti, Phys.Rev.D61,034503,034504 (2000).

15. J.M. Carmona, L. Del Debbio, M.D’Elia, A.Di Giacomo, B.Lucini, G. Paffuti, Phys.RevD66, 011503 (2002).

16. M. D’Elia, A. Di Giacomo, C. Pica, in preparation.

17. L. Del Debbio, M. D’Elia, A. Di Giacomo, B.Lucini, G.Paffuti, C.Pica, in preparation

18. M.E. Fischer,M.N. Barber, Phys. Rev. Lett.28, 1526 (1972).

19. E. Brezin , J. Physique 43, 15 (1982). 
Table 1.

The (pseudo) critical indices for weak 1st order and second order $O(4)$ phase transition.

\begin{tabular}{|c|c|c|c|c|}
\hline & $\alpha$ & $\gamma$ & $\nu$ & $y_{h}$ \\
\hline 1st order & 1 & 1 & $1 / 3$ & 3 \\
$O(4)$ 2nd order & $-.25(1)$ & $1.47(1)$ & $.75(1)$ & $2.49(1)$ \\
\hline
\end{tabular}

